\title{
Inhibition of E-cadherin/catenin complex formation by $O$-linked $N$-acetylglucosamine transferase is partially independent of its catalytic activity
}

\author{
HAIYAN LIU*, YUCHAO GU*, JIEQIONG QI, CUIFANG HAN, \\ XINLING ZHANG, CHUANLIN BI and WENGONG YU \\ Department of Glycobiology, School of Medicine and Pharmacy, \\ Ocean University of China, Qingdao, Shandong 266003, P.R. China
}

Received February 3, 2015; Accepted November 24, 2015

DOI: $10.3892 / \mathrm{mmr} .2015 .4718$

\begin{abstract}
. p120-catenin (p120) contains a large central armadillo repeat domain, via which it binds to E-cadherin to stabilize the latter, thereby regulating cell-to-cell adhesion. A previous study by our group demonstrated that O-linked $\mathrm{N}$-acetylglucosamine (O-GlcNAc) is involved in the regulation of the interaction between p120 and E-cadherin. As O-GlcNAc transferase (OGT) is able to directly bind to the majority of its target proteins, the present study hypothesized that OGT may additionally regulate the formation of the E-cadherin/catenin complex independent of its catalytic activity. To verify this hypothesis, a catalytically inactive OGT mutant was expressed in H1299 cells, and its effects on the formation of the E-cadherin/catenin complex were assessed. A cytoskeleton-binding protein extraction assay confirmed that OGT inhibited the formation of the E-cadherin/catenin complex independent of its catalytic activity. In addition, co-immunoprecipitation and pull-down assays were used to evaluate the interaction between OGT and p120. Immunoblotting indicated that OGT was able to directly bind to $\mathrm{p} 120$. To determine the domain of p120 involved in binding to OGT, a series of deletion mutants of p120 were constructed and subjected to protein binding assays by pull-down assays. Immunoblotting showed that OGT bound to the regulatory and armadillo domains of p120, which might interfere with the interaction between p120 and E-cadherin. Finally, OGT, p120 and E-cadherin cytoplasmic domains (ECD) were recombinantly expressed in BL21 (DE3) recombinant $E$. coli
\end{abstract}

Correspondence to: Professor Yuchao Gu, Department of Glycobiology, School of Medicine and Pharmacy, Ocean University of China, 5 Yushan Road, Qingdao, Shandong 266003, P.R. China E-mail: guych@126.com

${ }^{*}$ Contributed equally

Key words: p120, O-linked $N$-acetylglucosamine transferase, E-cadherin, E-cadherin/catenin complex, cytoskeleton cells, and a glutathione S-transferase (GST) pull-down assay was performed to assess the interactions among the purified recombinant proteins. Immunoblotting indicated that maltose-binding protein (MBP)-OGT inhibited the binding of His-p120 to GST-ECD in a dose-dependent manner. All of these results suggested that OGT inhibited the formation of the E-cadherin/catenin complex through reducing the interaction between p120 and E-cadherin. The present study provided a novel underlying mechanism of the regulation of the interaction between p120 and E-cadherin, and thus E-cadherin-mediated cell-cell adhesion, which has essential roles in cancer development and progression.

\section{Introduction}

Members of the classical cadherin family are transmembrane glycoproteins that mediate calcium-dependent homophilic adhesion via trans-dimerization of cadherin ectodomains between adjacent cells. They are essential for establishing tissue organization as well as maintaining tissue homeostasis, cell migration and wound healing (1). Among the large number of the classic cadherin family members, E-cadherin in epithelial tissues has been studied most extensively. The distal portion of the cadherin cytoplasmic tail binds to $\beta$-catenin, which in turn binds to $\alpha$-catenin and thereby links the adhesive complex to the actin cytoskeleton (2). Like $\beta$-catenin, p120-catenin (p120) binds to the cytoplasmic tail of all classic cadherins; however, p120 interacts with the juxtamembrane domain (JMD) to stabilize cadherin-catenin complexes at the cell surface (3-5). It has been demonstrated that a reduction in $\mathrm{p} 120$ protein levels or uncoupling of the p120-JMD interaction in cultured cells significantly increases the internalization of cadherin, thereby reducing the amount of cadherin available to facilitate cell-cell adhesion $(3,6)$. Therefore, p120 is a gatekeeper of cadherin turnover in vertebrates (7), and the balancing of the interaction between p120 and E-cadherin is an important underlying mechanism of the regulation of E-cadherin-mediated cell-cell adhesion.

Multiple mRNA variants of p120 exist, encoding different isoforms, which all consist of a large central armadillo repeat domain flanked by an N-terminal and a C-terminal region (8). 
This central domain has ten armadillo repeats, which is used to bind E-cadherin, and thus to stabilize E-cadherin and regulate cell-to-cell adhesion. The N-terminal domain of the longest p120 isoform (p120 1ABC) contains a coiled-coil domain and a regulatory phosphorylation domain. The phosphorylation domain comprises an abundance of Ser, Thr and Tyr residues, which are phosphorylated under certain conditions. The phosphorylation of p120 is involved in protein-protein interactions and the regulation of cell adhesion (9-11). The C-terminal region contains potential nuclear localization and export signals.

$N$-acetylglucosamine transferase (OGT) catalyzes the $\mathrm{O}-\mathrm{GlcNAcylation}$ of proteins, which is an O-linked glycosylation involving attachment of $\beta$-GlcNAc to Ser/Thr residues, and whose removal is catalyzed by O-GlcNAcase (OGA). A previous study by our group demonstrated that O-GlcNAc enhanced breast cancer metastasis, which was mainly caused by the decrease of cell surface E-cadherin. Moreover, p120 was shown to be modified by O-GlcNAc, which was likely to be involved in the regulation of the E-cadherin/catenin complex (12).

As OGT has been demonstrated to be able to directly bind to the majority of its target proteins, the present study hypothesized that OGT may additionally regulate the formation of the E-cadherin/catenin complex independent of its catalytic activity. To verify this hypothesis, a catalytically inactive OGT mutant was expressed in H1299 cells, and its effects on the formation of the E-cadherin/catenin complex were assessed. In addition, the interaction between OGT and p120 was analyzed by co-immunoprecipitation and pull-down assays. Furthermore, a series of deletion mutants of p120 were constructed to determine the domain of p120 binding to OGT. Finally, a pull-down assay was performed to evaluate the role of OGT in the interaction between purified recombinant p120 and ECD. This interaction may represent a novel underlying mechanism of the regulation of the interaction between p120 and E-cadherin, and thus E-cadherin-mediated cell-cell adhesion, which has essential roles in cancer development and progression.

\section{Materials and methods}

Cell lines and culture. HEK293T human embryonic kidney cells (Baili Biological Technology Co., Ltd., Shanghai, China) were cultured in Dulbecco's modified Eagle's medium (DMEM; Lonza Group, Ltd., Basel, Switzerland) containing high glucose and $10 \%(\mathrm{v} / \mathrm{v})$ fetal calf serum (FBS; Gibco, Thermo Fisher Scientific, Inc., Waltham, MA, USA). The H1299 human lung carcinoma cells (Baili Biological Technology Co., Ltd., Shanghai, China) with low E-cadherin expression was cultured in RPMI-1640 medium (Gibco; Thermo Fisher Scientific, Inc.) supplemented with 10\% FBS. The 4T1 cell line was kindly provided by Professor Fred R. Miller prior to 2006 (Department of Oncology, Barbara Ann Karmanos Cancer Institute, Wayne State University, Detroit, MI, USA), which is derived from a mammary tumor that arose spontaneously in a wild-type (wt) BALB/c mouse (13). 4T1 cells were cultured in DMEM supplemented with $10 \%$ FBS, $1 \mathrm{mM}$ mixed non-essential amino acids and $2 \mathrm{mM}$ L-glutamine. The generation of OGT-silenced 4T1 cells was performed as reported previously (12). Cells were maintained in a humidified atmosphere containing $5 \% \mathrm{CO}_{2}$ at $37^{\circ} \mathrm{C}$. To elevate the global levels of O-GlcNAc, cells were treated with $10 \mu \mathrm{M}$ Thiamet-G (Sigma-Aldrich, St. Louis, MO, USA), a selective OGA inhibitor, for $24 \mathrm{~h}$. The A549 human lung carcinoma cell line was cultured in F12K medium supplemented with 10\% FBS. Lipofectamine 2000 (Invitrogen; Thermo Fisher Scientific, Inc.) was used for cell transfection according to the manufacturer's instructions.

Plasmid construction. A cytomegalovirus (CMV) vector expressing Flag-tagged human nuclear/cytoplasmic (nc)OGT (pCMV-Flag-OGT) was kindly provided by Professor Jin Won Cho (Department of Integrated OMICS for Biomedical Science, Yonsei University, Seoul, South Korea) (14). A vector expressing a Flag-tagged human mutant OGT, H558A (His558 to Ala), was constructed by site-directed mutagenesis. The full-length sequence of the expression vector of mutant OGT was amplified by polymerase chain reaction (PCR) amplification of the pCMV-Flag-OGT plasmid, using Phusion High-Fidelity DNA Polymerase (Thermo Fisher Scientific), a Juhao JH-9600 PCR machine (Taizhou Juhao Import and Export Co., Ltd., Taizhou, China) and the following primers: Forward, 5'-TGCTCCTACTTCTCACCTTATGCA-3' and reverse, 5'-TTCCCAAAGTCGGAACTCAC-3'. The PCR product was phosphorylated and then self-ligated to obtain the vector pCMV-Flag-OGT H558A by using a Blunting Kination Ligation kit (Takara, Otsu, Japan). Maltose-binding protein (MBP)-tagged OGT-expressiong vector (pMAL-c2X-OGT) was a generous gift from Professor David J. Vocadlo (Department of Molecular Biology and Biochemistry, Simon Fraser University, Burnaby, BC, Canada) (15). A pGEX-2T-ECD (pGEX-ECD) vector expressing the glutathione S-transferase-tagged complete E-cadherin cytoplasmic domain (GST-ECD), including the JMD domain, was obtained as described as previously (12). A linker (5'-AAGCTTACCATG GACTACAAGGACGACGATGACAAGGGATCCGGTGGT ACCGCGGCCGCTGATATCTGATCTAGA-3'), containing a Flag tag coding sequence and multiple restriction enzyme sites, was inserted between the HindIII and $\mathrm{XbaI}$ sites of a pcDNA3.1 vector, creating a vector named pcDNA3-Flag. The full-length coding region of p120 was amplified by PCR from the LZRS-murine p120 1A-neo vector (obtained from Professor Albert Reynolds, Vanderbilt University Medical Center, Nashville, TN, USA), using the forward primer 5'-GGT ACCATGGACGACTCAGAG-3' carrying a KpnI site at the 5' end and the reverse primer 5'-GATATCCTAAATCTTCTG CATCAA-3' with an EcoRV site at the 5' end. The DNA fragment was cut using restriction endonucleases $K p n \mathrm{I}$ and EcoRV, and ligated into the pcDNA3-Flag vector, resulting in an N-terminal Flag-fused p120 expression vector named pcDNA3-Flag-p120. The PCR product was also cloned into the pET30a vector using the same restriction sites as above, and the His-tagged p120 expression vector pET30a-p120 was obtained.

To construct p120 mutants, DNA fragments encoding amino acids 1-101 at the N-terminus of p120 (F1), amino acids $102-323$ at the regulatory domain (F2), amino acids 324-832 encompassing the armadillo repeat domain (F3) and amino acids 833-933 at the C-terminus (F4), respectively, 
were amplified by PCR using the corresponding primers: F1 forward, 5'-GGTACCATGGACGACTCAGAG-3' and reverse, 5'-GATATCTCACCTGGGGATAGTGCT-3'; F2 forward, 5'-GGTACCATGCAGGAGCCAG-3' and reverse, 5'-GATATC TCAGTCTTCATAGCTCCTGAG-3'; F3 forward, 5'-GGT ACCATGATTGGTGAAGAGG-3' and reverse, 5'-GATATC TCATGGCTTCCGAAGCTC-3'; F4 forward, 5'-GGTACC CTGGAAAAAGAAGG-3' and reverse, 5'-GATATCCTA AATCTTCTGCATCAA-3'. The 5' ends of all forward primers contain the KpnI site and the $5^{\prime}$ ends of all reverse primers contain the EcoRV site. The PCR products were cut using $K p n \mathrm{I}$ and EcoRV. The fragment between BamHI and EcoRI in the pGEX-2T vector (Addgene; Cambridge, MA, USA) containing the GST tag was replaced by a linker (5'-GGA TCCGGTGGTACCGCTAGCGCGGCCGCTCCCGGGGAA TTC-3') containing restriction sites for BamHI, KpnI, NheI, NotI, SmaI and EcoRI, followed by digestion with KpnI and SmaI. The digested PCR products were cloned into the modified pGEX-2T vector. A total of four p120 mutant expression vectors, pGEX-F1, pGEX-F2, pGEX-F3 and pGEX-F4, were obtained.

The coding sequences for GST-fused p120 mutants were amplified by PCR using the fixed forward primer 5'-GAATTC ATGTCCCCTATACTAGGTT-3' carrying an EcoRI restriction site at the $5^{\prime}$ end and one of the corresponding reverse primers (F1, F2, F3 and F4 reverse) as described above, and the vectors of pGEX-F1, pGEX-F2, pGEX-F3 and pGEX-F4, respectively, were used as templates. The PCR products were cloned into the EcoRI and EcoRV sites of pcDNA3.1 and the resulting vectors were named as pcDNA3-GST-p120 mutants. The constructed vectors were transfected into 4T1 mammary cancer cells to express GST-fused p120 mutants. All primers and nucleotide linkers were purchased from BGI (Shanghai, China). All restriction enzymes were purchased from Takara Bio, Inc. (Otsu, Japan) and were used according to the manufacturer's instructions.

Protein expression and purification. pET30a-p120, pGEX-ECD, pMal-c2X-OGT, pGEX-p120 mutants and negative control vectors pMal-c2X and pGEX-2T were individually transfected into BL21 (DE3) Escherichia (E.) coli cells (Novagen, Inc., Madison, WI, USA) to obtain the proteins: His tagged p120 (His-p120), GST-tagged ECD (GST-ECD), MBP-tagged OGT (MBP-OGT), GST-tagged F1-F4 (GST-F1, GST-F2, GST-F3, GST-F4), MBP and GST. Single clones were selected in the presence of $100 \mu \mathrm{g} / \mathrm{ml}$ ampicillin (pGEX-2T and pMal-c2X systems) or $50 \mu \mathrm{g} / \mathrm{ml}$ kanamycin (pET30a system). All selected clones were grown in Lysogeny broth containing antibiotics (kanamycin or ampicillin) at $37^{\circ} \mathrm{C}$ to an optical density at $600 \mathrm{~nm}$ of 0.6 , followed by addition of $0.2 \mathrm{mM}$ isopropyl-1- $\beta$-D-thiogala ctopyranoside (Invitrogen; Thermo Fisher Scientific, Inc.) and incubation at room temperature overnight. Cells were harvested and lysed by sonication $(300 \mathrm{~W}, 80 \mathrm{~Hz}, 15 \mathrm{~min})$ and the lysate was cleared by centrifugation $(12,000 \mathrm{x} g$ for $30 \mathrm{~min})$. The respective proteins were purified using HisTrap HP (GE Healthcare, Little Chalfont, UK), glutathione sepharose 4B (GS4B) beads (GE Healthcare) or amylose resin (New England BioLabs, Ipswich, MA, USA) following the manufacturer's protocols.
pcDNA3-Flag-p120, pCMV-Flag-OGT and pcDNA3-GST-p120 mutants were transfected into HEK293T cells for $48 \mathrm{~h}$, followed by purification of the proteins using anti-Flag antibody agarose (Abmart, Berkeley Heights, NJ, USA) or GS4B beads. OGT-silenced 4T1 cells mediated by the pLKO.1-small hairpin OGT (pLKO.1-shOGT) lentiviral vector and selected with $8 \mu \mathrm{g} / \mathrm{ml}$ puromycin for 2 weeks, were stored in our laboratory.

Antibodies.Immunoblotting and immunoprecipitation(IP)were performed with the following primary antibodies: Polyclonal rabbit anti-OGT (1:500; O6264; Sigma-Aldrich), monoclonal mouse anti-GST (1:1,000; sc-138; Santa Cruz Biotechnology, Inc., Dallas, TX, USA), monoclonal mouse anti- $\beta$-catenin (1:1,000; sc-7963; Santa Cruz Biotechnology, Inc.), polyclonal rabbit anti-p120 (1:1,000; sc-1101; Santa Cruz Biotechnology, Inc.), monoclonal mouse anti-glyceraldehyde-3-phosphate dehydrogenase (GAPDH; 1:5,000; sd-137179; Santa Cruz Biotechnology, Inc.), polyclonal rabbit anti- $\beta$-actin (1:5,000; sc-130657; Santa Cruz Biotechnology, Inc.), monoclonal mouse anti-O-GlcNAc (1:2,000; ab24687; Abcam, Cambridge, MA, USA), polyclonal rabbit anti-His-tag $(1: 1,000 ; 2365 \mathrm{~S}$; Cell Signaling Technology, Inc., Danvers, MA, USA), monoclonal mouse anti-Flag-tag (1:5,000; M20008; Abmart) and monoclonal mouse anti-E-cadherin $(1: 5,000 ; 610181$; BD Biosciences, Franklin Lakes, NJ, USA).

$I P$, pull-down and immunoblot (IB) analyses. For immunoprecipitation, cells were lysed in radioimmunoprecipitation assay (RIPA) buffer [25 mmol/1 Tris- $\mathrm{HCl}$ (pH 7.4), $150 \mathrm{mmol} / \mathrm{l}$ $\mathrm{NaCl}, 1 \%$ Nonidet-P40, $0.1 \%$ sodium dodecyl sulfate (SDS), $1 \mathrm{mmol} / 1$ ethylenediaminetetraacetate (EDTA), $1 \mathrm{mmol} / 1$ $\mathrm{Na}_{3} \mathrm{VO}_{4}$ and $10 \mathrm{mmol} / \mathrm{l} \mathrm{NaF}$ ] containing a protease inhibitor cocktail (Roche, Basel, Switzerland), $5 \mu \mathrm{M}$ glycosidase inhibitor PUGNAc (Toronto Research Chemicals Inc., Toronto, OT, Canada) and $10 \mathrm{mM}$ streptozotocin (STZ; Sigma-Aldrich); for co-immunoprecipitation, the cell lysate in RIPA buffer was diluted two-fold with phosphate-buffered saline (PBS). The indicated antibodies were added, followed by incubation overnight at $4^{\circ} \mathrm{C}$. Subsequently, protein A/G agarose beads (Abmart) were added, followed by incubation for another hour at $4^{\circ} \mathrm{C}$. Beads were collected by centrifugation $(300 \mathrm{x} \mathrm{g}$ for $3 \mathrm{~min}$ at $4^{\circ} \mathrm{C}$ ) and washed three times with binding buffer [50 mM Tris- $\mathrm{HCl}$, pH 7.3, $150 \mathrm{mM} \mathrm{NaCl}, 3 \mathrm{mM} \mathrm{MgCl}{ }_{2}, 1 \mathrm{mM}$ EDTA, $1 \mathrm{mM}$ dithiothreitol, and $0.1 \%$ (w/v) Triton X-100, $1 \mathrm{mM} \mathrm{Na} \mathrm{VO}_{4}, 10 \mathrm{mM} \mathrm{NaF}$ and protease inhibitor cocktail]. The collected beads were boiled in $50 \mu 1$ of SDS loading buffer and detected by IB analysis.

For the pulldown assay, the indicated proteins were incubated in the binding buffer (final volume, $200 \mu \mathrm{l}$ ) for $45 \mathrm{~min}$ at room temperature. The protein complexes were then isolated by incubation with GS4B beads or anti-Flag antibody agarose beads. After $30 \mathrm{~min}$ of gentle agitation, beads were collected by centrifugation and washed three times with binding buffer.

IB was performed according to established protocols and developed using enhanced chemiluminescence reagent (GE Healthcare). In brief, the cell supernatants were recovered, separated by $12 \%$ SDS-polyacrylamide electrophoresis (Shanghai Bogoo Biotechnology, Co., Ltd., Shanghai, China) and transferred onto a $0.45 \mu \mathrm{M}$ polyvinylidene difluoride 
membrane (EMD Millipore, Billerica, MA, USA). Following blocking the membrane with Tris-buffered saline-Tween-20 containing $5 \%$ non-fat milk for $1 \mathrm{~h}$ at room temperature, the membrane was incubated with the following primary antibodies: Anti-OGT, anti-GST, anti- $\beta$-catenin, anti-p120, anti-GAPDH, anti- $\beta$-actin, anti-O-GlcNAc, anti-His-tag, anti-Flag-tag, and anti-E-cadherin antibodies overnight at $4^{\circ} \mathrm{C}$. The membrane was incubated with horseradish peroxidase (HRP)-conjugated goat anti-mouse IgG (1:5,000; 7076; Cell Signaling Technology, Inc.) or HRP-conjugated goat anti-rabbit IgG (1:5,000; 7074; Cell Signaling Technology, Inc.) for $1 \mathrm{~h}$ at room temperature and the bands were detected using the SignalFire ${ }^{\mathrm{TM}}$ Enhanced Chemiluminescence reagent (Cell Signaling Technologies, Inc.) in a dark room.

The experiment was repeated a minimum of three times and the images shown are representative of the three repetitions.

Extraction of cytoskeleton-binding proteins. To separate cytoskeleton-binding proteins from other cell constituents, H1299 and 4T1 cells were washed twice in PBS, lysed on ice for $15 \mathrm{~min}$ in Triton X-100 lysis buffer [300 mM sucrose, $10 \mathrm{mM}$ piperazine- $N, N$ '-bis(2-ethanesulfonic acid) $(\mathrm{pH} 6.8), 50 \mathrm{mM}$ $\mathrm{NaCl}, 3 \mathrm{mM} \mathrm{MgCl} 2,0.5 \%$ Triton X-100, $0.1 \mathrm{mg} / \mathrm{ml}$ DNase I, $0.1 \mathrm{mg} / \mathrm{ml}$ RNase A, $1.2 \mathrm{mM}$ phenylmethanesulfonyl fluoride, the protease inhibitor cocktail, $5 \mu \mathrm{M}$ glycosidase inhibitor PUGNAc and $10 \mathrm{mM}$ STZ], passed through a 26-gauge needle (Beckman Coulter, Shanghai, China) four times and then subjected to centrifugation at $48,000 \mathrm{x}$ g for $10 \mathrm{~min}$ at $4^{\circ} \mathrm{C}$. The pellet was solubilized in loading buffer containing $10 \%$ SDS, and the supernatant and pellet fractions were loaded on separate gels (Shanghai Bogoo Biotechnology, Co., Ltd.) and processed as above (16).

The experiment was repeated a minimum of three times and the images shown are representative of the three repetitions.

\section{Results}

OGT inhibits the formation of the E-cadherin/catenin complex. A previous study by our group demonstrated that O-GlcNAc is involved in the regulation of the interaction between p120 and E-cadherin (12). As OGT was able to directly bind to most of its target proteins, the present study hypothesized that OGT may also regulate the formation of the E-cadherin/catenin complex independent of its catalytic activity. To verify this hypothesis, catalytically inactive OGT mutant was expressed in H1299 cells, and its effects on the formation of the E-cadherin/catenin complex were assessed. For this, H558A, a His558-to-Ala mutant of OGT was generated, which is known to be void of any catalytic activity (17). An immunoblotting assay indicated that the expression of wtOGT resulted in a marked increase in global cellular O-GlcNAc levels, while H558A only slightly increased O-GlcNAc levels (Fig. 1A). A Co-IP assay showed that in endogenous OGT-silenced cells, the interaction of p120 with wtOGT was similar to that with or H558A (Fig. 1B). Endogenous OGT was not detectable by anti-OGT antibody in OGT-silenced cells, which excluded the possibility that endogenous OGT associated with p120 or heterooligomerized with H558A. These results indicated that H558A was suitable for use in experiments aiming to elucidate the effect of OGT protein on the formation of the E-cadherin/p120 complex.

As a previous study demonstrated that the binding of the E-cadherin/catenin complex to the cytoskeleton is essential for strong cell-cell adhesion (18), the present study investigated the effects of OGT on the formation of the E-cadherin/catenin/cytoskeleton complex. wtOGT or H558A expression vectors were transfected into H1299 cells, followed by isolation of the E-cadherin/catenin complex as the Triton X-100-insoluble fraction. Immunoblotting demonstrated that the expression of H558A mutant inhibited the formation of the E-cadherin/catenin complex to a similar extent to wtOGT (Fig. 1C), which suggested that OGT may also regulate the formation of the E-cadherin/catenin complex independent of its catalytic activity. By detection of GAPDH, which is not able to bind to the cytoskeleton, it was confirmed that the extracted E-cadherin adhesion complex was not contaminated by any Triton X-100-soluble proteins. $\beta$-Actin was detected as a loading control.

To verify the above findings, Thiamet-G was used to inhibit OGA activity and thereby increase O-GlcNAc levels in OGT-knockdown 4T1 cells. A previous study by our group proved that the elevation of O-GlcNAc by Thiamet-G effectively reduced the amount of cytoskeleton-associated E-cadherin compared with that in control cells (12). As shown in Fig. 1D, OGT silencing reduced the global $\mathrm{O}-$ GlcNAc levels and markedly increased the amount of cytoskeleton-associated E-cadherin; however, Thiamet-G treatment did not obviously decrease the amount of cytoskeleton-associated E-cadherin, while it partly restored the global O-GlcNAc levels in the OGT-silenced 4T1 cells. These results implied that OGT protein itself was able to modulate cytoskeleton-associated E-cadherin, independent of its catalytic activity.

These results suggested that OGT directly bound to certain proteins which are relevant to the function of E-cadherin, and thus inhibited the formation of the E-cadherin adhesion complex.

OGT binds to p120. A previous study by our group found that p120 was modified by O-GlcNAc (12). To clarify whether OGT directly binds to p120, co-IP and pull-down assays were performed. As the available anti-p120 antibody was inefficient in co-IP assays, the anti-OGT antibody was used for co-IP of endogenous proteins in 4T1 cells. The results indicated that p120 was able to be co-precipitated with OGT (Fig. 2A). As the transfection efficiency was low for 4T1 cells, the expression vectors of Flag-OGT and Flag-p120 were individually transfected into HEK293T cells, which were then subjected to co-IP assays using the anti-Flag antibody to verify the interaction between OGT and p120. IB analysis showed that OGT was able to be co-precipitated with p120 (Fig. 2B), and vice versa (Fig. 2C). In addition, a pull-down assay was performed to determine the interaction between p120 and OGT. GST and GST-p120 were recombinantly expressed in BL21 (DE3) E. coli cells and purified, and Flag-OGT was immunopurified from HEK293T cells transfected with plasmid encoding Flag-OGT by using anti-Flag antibody agarose. Purification was performed under harsh conditions (using RIPA buffer) to exclude the co-purification 
A Ctrl wtOGT H558A

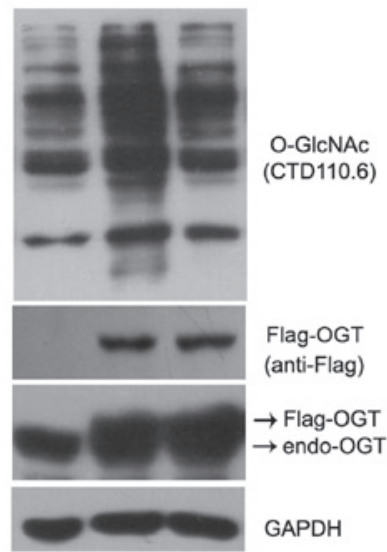

C

Triton X-100 insoluble Triton X-100 soluble Ctrl wtOGT H558A Ctrl wtOGT H558A

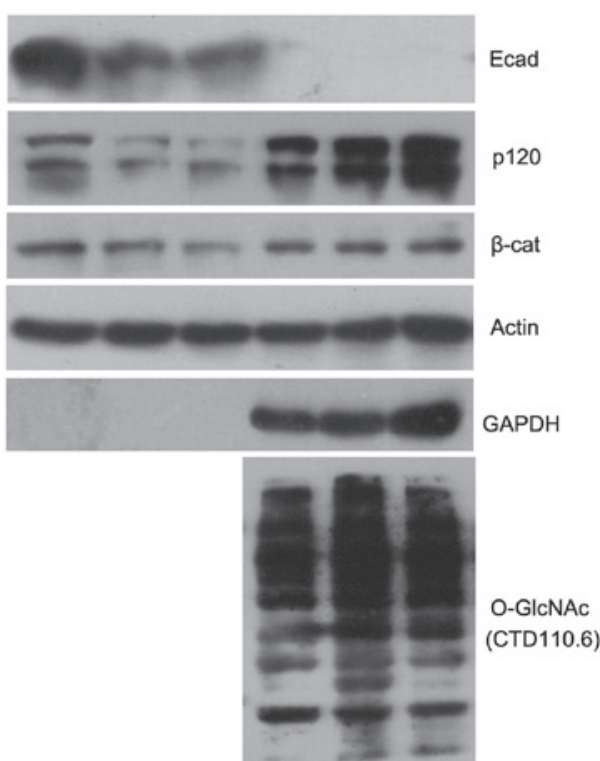

$\mathbf{B}$

B $\quad$ shOGT

Ctrl wtOGT H558A

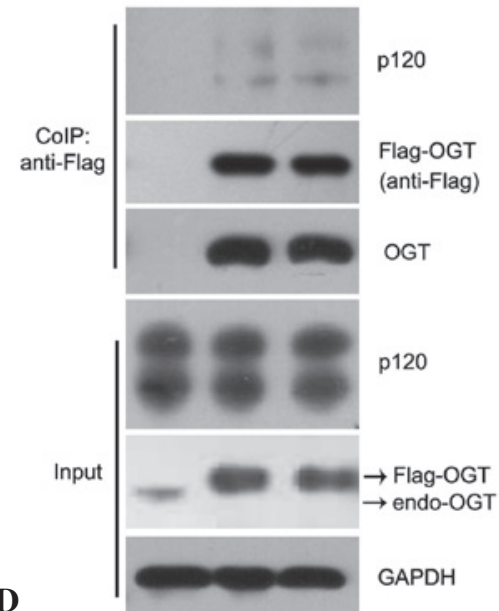

Triton X-100 insoluble Triton X-100 soluble

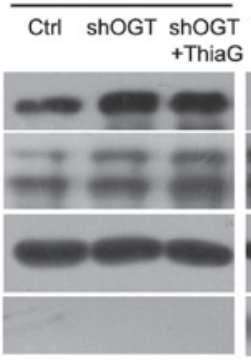
T +ThiaG

Ecad

p120

Actin

GAPDH

O-GICNAC

(CTD110.6)

Figure 1. OGT inhibits the formation of E-cadherin adhesion complex. (A-C) The Ctrl, Flag-tagged wtOGT and mutant OGT H558A were respectively transfected into H1299 cells for 48 h, which were subjected to analysis. (A) Total cellular O-GlcNAc as well as exogenous and endogenous Flag-OGT levels were determined by immunoblotting with GAPDH detected as an internal standard. (B) wtOGT and H558A plasmids were respectively transfected into OGT-silenced cells, and empty plasmid-transfected cells served as the control. Cells were subjected to CoIP with anti-Flag antibody agarose. The precipitated Flag-OGT was immunoblotted with anti-Flag, anti-OGT or anti-p120 antibodies, and immunoblotting for p120 was performed. Input Flag-OGT and endo-OGT were immunoblotted by anti-OGT antibody as indicated by the arrows. (C) Cytoskeleton-binding proteins from cells of various transfection groups (Ctrl, wtOGT and H558A) were obtained as the Triton X-100-insoluble fraction and subjected to immunoblot detection of E-cadherin, p120, $\beta$-catenin, $\beta$-actin and GAPDH alongside the Triton X-100-soluble protein fraction. $\beta$-Actin and GAPDH were detected as loading controls and internal references. O-GlcNAc antibody CTD110.6 was used to detect the global O-GlcNAc levels. (D) Control and OGT-silenced 4T1 cells were treated with or without selective O-GlcNAcase inhibitor Thiamet-G, and the cytoskeleton-binding proteins (Triton X-100-insoluble) and supernatant fractions (Triton X-100-soluble) were subjected to immunoblotting with antibodies against E-cadherin, p120, $\beta$-actin, GAPDH and O-GlcNAc. Immunoblotting with CTD110.6 revealed the differences in O-GlcNAc levels among the three samples. O-GlcNAc, O-linked $N$-acetylglucosamine; OGT, O-GlcNAc transferase; Ctrl, empty plasmid; wt, wild-type; H558A, mutant OGT; endo, endogenous; shOGT, small-hairpin RNA specific for OGT; $\beta$-cat, $\beta$-catenin; Ecad, E-cadherin; ThiaG, Thiamet-G; CoIP, co-immunoprecipitation; GAPDH, glyceraldehyde-3-phosphate dehydrogenase.

of p120-binding molecules. Flag-OGT immobilized to anti-Flag antibody agarose was incubated with GST or GST-p120, and a subsequent immunoblotting assay indicated that GST-p120 but not GST was able to bind to Flag-OGT (Fig. 2D). These results demonstrated that OGT was able to bind to p120. In addition, a pull-down assay demonstrated that OGT mutant H558A was able to bind to p120 in a similar manner to that of wtOGT (Fig. 2E).
OGT binds to the regulatory and armadillo domains of p120. In order to identify the OGT-binding domain in p120, several deletion mutants of p120 were constructed, as illustrated in Fig. 3. It was first attempted to express p120 deletion mutants as Flag-tagged proteins in HEK293T cells; however, the expression levels of certain mutants were very low (data not shown). To overcome this shortcoming, the mutants were fused to GST and effectively expressed in HEK293T 
A

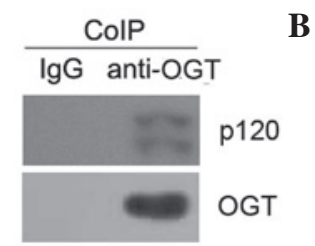

D

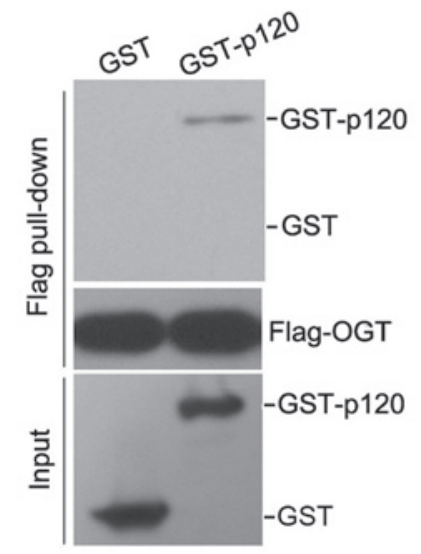

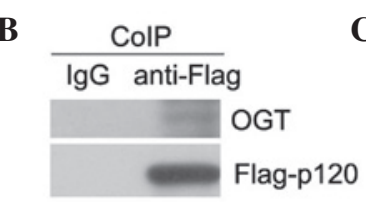

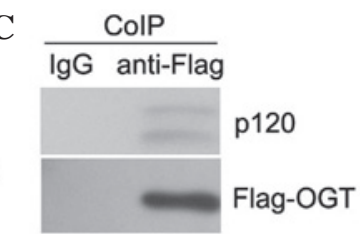

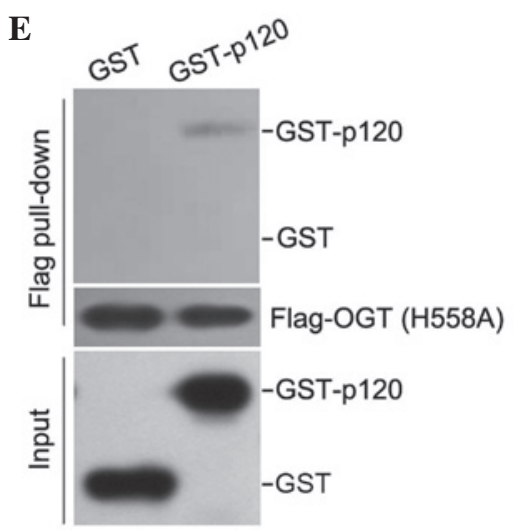

Figure 2. OGT binds to p120. (A) Rabbit OGT antibody was used for CoIP of endogenous proteins. (B) Flag-p120 or (C) Flag-OGT was overexpressed in HEK293T cells and the anti-Flag antibody was used for CoIP assays. IgG was used as a negative control. Anti-OGT and anti-p120 antibodies were respectively used for immunoblotting of the co-precipitated OGT and p120. (D) For the in vitro pull-down assay of p120 and OGT, GST and GST-p120 were recombinantly expressed and purified from BL21 (DE3) Escherichia coli cells with GS4B beads, and Flag-OGT was immunopurified from HEK293T cells by using anti-Flag antibody agarose, followed by incubation with GST or GST-p120, respectively. GST was used as a negative control. OGT and the bound p120 were detected using anti-Flag or anti-GST antibodies. (E) Pull-down assay of p120 and OGT mutant H558A performed as above. OGT, O-linked $\mathrm{N}$-acetylglucosamine transferase; H558A, mutant OGT; IgG, immunoglobulin G; CoIP, co-immunoprecipitation; GST, glutathione S-transferase.

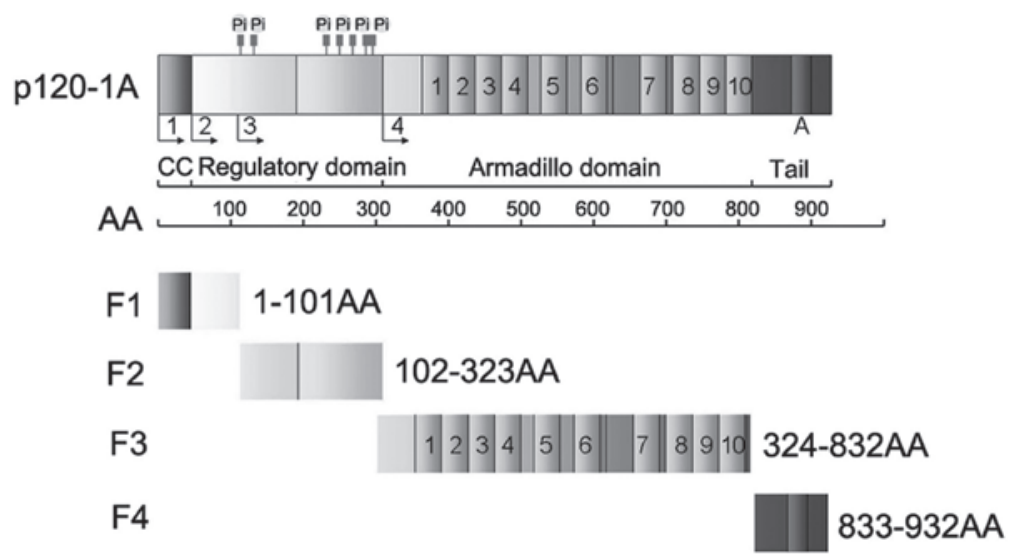

Figure 3. Construction of p120 deletion mutants. Four alternative translation initiation sites within the N-terminal globular domain are shown as F1-F4. The central part of p120 contains 10 armadillo repeats. One alternatively spliced exon A resides within the C-terminal domain. The N-terminal domain of p120 contains a CC domain and a regulatory phosphorylation domain. The phosphorylation domain comprises a large amount of Ser, Thr and Tyr residues, which are phosphorylated under certain conditions. The phosphorylation of p120 is involved in protein-protein interactions and the regulation of cell adhesion. This Armadillo domain binds with E-cadherin, thus stabilizing E-cadherin and regulating cell-to-cell adhesion. The C-terminal region is usually associated with potential nuclear localization and export signaling. The p120-1A coding sequence was divided into four segments, which were attached to the N-terminal glutathione S-transferase-tag and cloned into pcDNA3 or pGEX-2T vectors. AA, amino acid; CC, coiled coil; Pi, phosphate.

cells. GS4B agarose beads were used to precipitate the GST fusion proteins from the cell lysate. An immunoblot assay demonstrated that OGT interacted with the GST-tagged F2 and F3 mutants but not with GST-tagged F1 and F4 mutants (Fig. 4A). In another experiment, GST-tagged p120 mutants were overexpressed and purified from BL21 (DE3) recombinant $E$. coli cells, bound to GS4B agarose and incubated with HEK293T-cell lysate. Consistent with the above results, OGT was able to efficiently bind to GST-tagged F2 and F3 mutants (Fig. 4B).
To exclude the possibility that OGT binds to the regulatory and armadillo domains of p120 depending on the participation of other protein partners, an OGT binding assay was performed. Flag-OGT from HEK293T cells was immobilized to anti-Flag antibody agarose and washed with a harsh lysis buffer in order to inhibit any protein-protein interactions, followed by incubation with an equal amount of the respective GST-tagged p120 mutants, which were overexpressed and purified from BL21 (DE3) recombinant $E$. coli cells. A Flag pull-down assay showed that only GST-tagged F2 and F3 

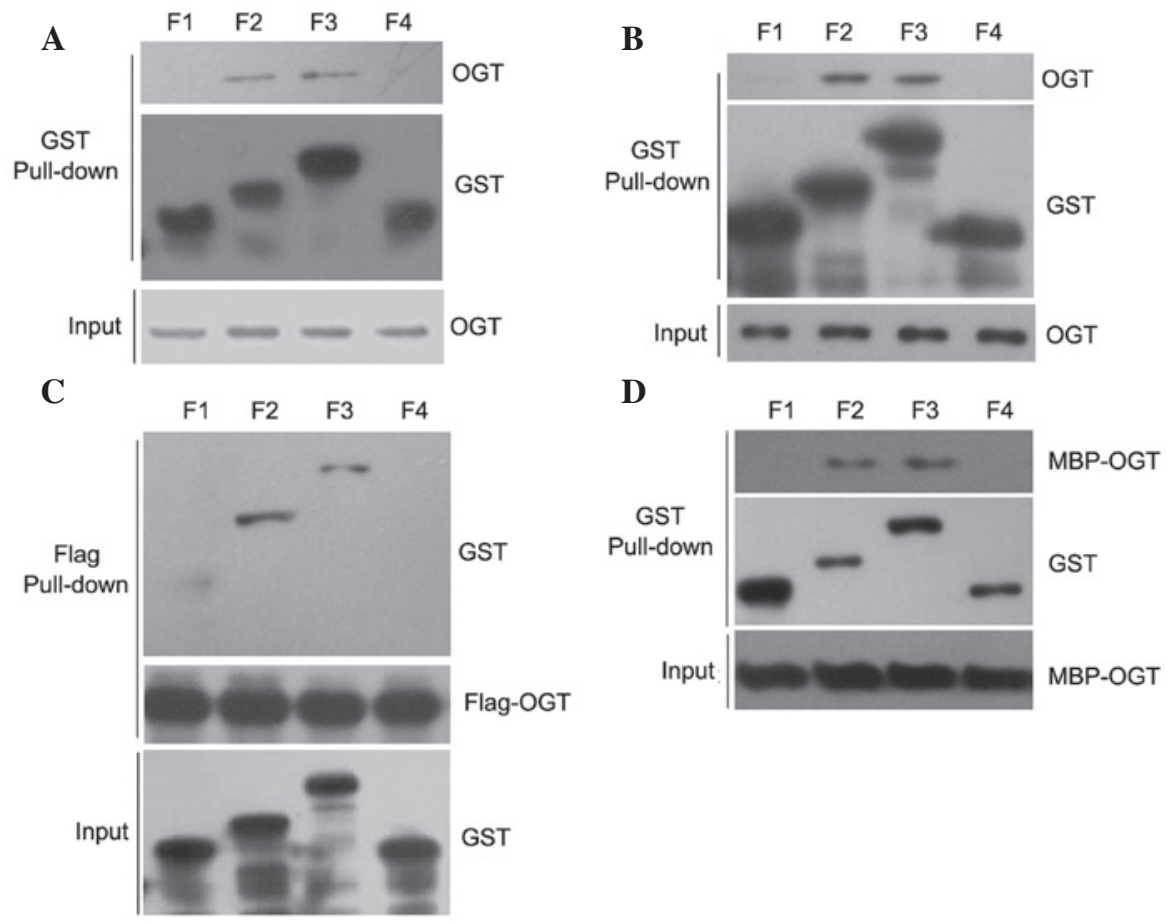

Figure 4. OGT binds to the regulatory and armadillo domains of p120, F1-F4. (A) GST-fused p120 mutants F1-F4 were expressed in HEK293T cells. GS4B agarose beads were used to precipitate the GST fusion proteins from the cell lysate. The bound OGT at the top or GST-fused p120 mutants were immunoblotted using anti-OGT or anti-GST antibodies. (B) The GST-p120 mutants purified from BL21 (DE3) recombinant E. coli cells were immobilized on GS4B agarose beads, which were then incubated with HEK293T cell lysate, and the bound OGT was detected by immunoblotting with anti-OGT antibody as shown in the top panel. GST-p120 mutants were detected by using anti-GST antibody. (C) Flag-OGT, purified from HEK293T cells, was immobilized to anti-Flag antibody agarose, followed by incubation with GST-p120 mutants purified from BL21 (DE3) recombinant $E$. coli cells. Anti-Flag and anti-GST antibodies were used for immunoblotting of Flag-OGT and the bound GST-p120 mutants as shown in the top panel. (D) The GST-p120 mutants purified from BL21 (DE3) recombinant E. coli cells were immobilized to GS4B agarose beads and were then incubated with MBP-tagged OGT (MBP-OGT) purified from BL21 (DE3) recombinant E. coli cells. Anti-GST and anti-OGT antibodies were used for detecting GST-p120 mutants and the bound MBP-OGT. Lanes F1-F4: Cells transfected with plasmids containing the mutant fragments of the p120-1A coding sequence. E. coli, Escherichia coli; MBP, maltose-binding protein; OGT, O-linked $\mathrm{N}$-acetylglucosamine transferase; GST, glutathione S-transferase.

mutants were able to bind to Flag-OGT (Fig. 4C). In another experiment, equal amounts of MBP-OGT and GST-p120 mutants, which had been purified from the corresponding BL21 (DE3) recombinant $E$. coli cells, were co-incubated. Protein immobilized to GS4B agarose beads was then subjected to a GST pull-down assay with an identical result to that of the above assay (Fig. 4D). These results demonstrated that OGT was able to directly bind to regulatory as well as armadillo domains of p120. Considering that the armadillo domain of p120 was crucial for the binding of E-cadherin, the above findings suggested that OGT inhibited the interaction between p120 and E-cadherin.

OGT inhibits the interaction between p120 and E-cadherin. To elucidate whether OGT was able to inhibit the binding of p120 to E-cadherin, a GST pull-down assay was performed (Fig. 5). GST, GST-ECD, MBP, MBP-OGT and His-p120 were all purified from the corresponding BL21 (DE3) recombinant $E$. coli cells and the given recombinant proteins were incubated at the indicated doses. GST and MBP were used as negative controls in the GST pull-down assays in order to exclude the potential interference of GST and MBP tags. As shown in Fig. 5A, MBP-OGT inhibited the binding of His-p120 to GST-ECD in a dose-dependent manner, and MBP-OGT was not able to bind to GST-ECD in the presence or absence of p120. The results suggested that p120 was not able to interact with OGT and E-cadherin simultaneously, indicating that OGT and E-cadherin may compete for p120 binding. To verify this hypothesis, the present study examined whether OGT was able to bind to E-cadherin in cultured cells. A549 cells, which exhibit high endogenous E-cadherin expression, were subjected to a co-IP assay with anti-E-cadherin antibody. The results indicated that $\mathrm{p} 120$ and $\beta$-catenin, but not OGT, were able to bind to E-cadherin, or rather that the co-precipitated E-cadherin-bound p120 did not bind with OGT (Fig. 5B). Therefore, it was speculated that E-cadherin was not able to bind to p120 when the latter was bound to OGT, and vice versa. In conclusion, these results suggested that the binding of OGT to p120 may be the underlying molecular mechanism of the regulatory role of OGT on the formation of the E-cadherin/catenin complex, independent of its catalytic activity.

\section{Discussion}

E-cadherin is expressed mainly in epithelial cells and has a fundamental role in establishing and maintaining homotypic cell-cell adhesion, thereby contributing to normal development, epithelial cell polarity and carcinoma progression (19). The formation and stabilization of adhesion complexes are dynamic and highly regulated processes $(20,21)$. Endocytosis is a major regulatory mechanism for E-cadherin dynamics. 
A

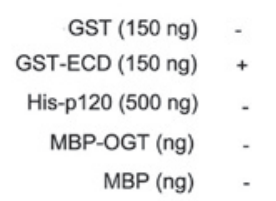

$\begin{array}{cc}- & + \\ + & - \\ - & + \\ 500 & - \\ - & -\end{array}$

B

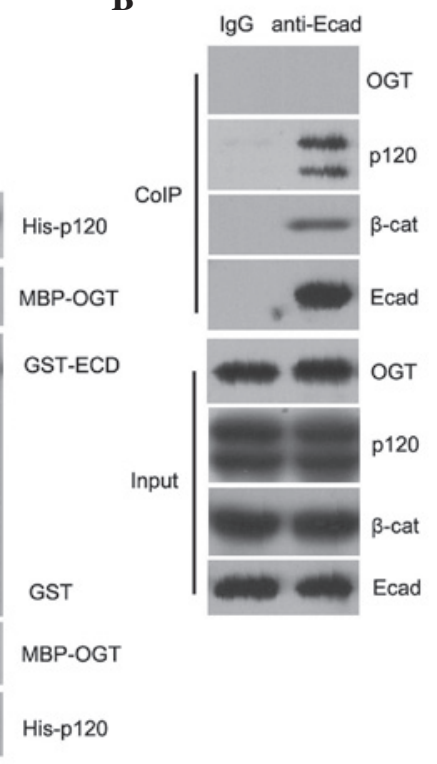

Figure 5. OGT inhibits the interaction between p120 and E-cadherin. (A) The purified GST, MBP, MBP-OGT, His-p120 or GST-ECD from BL21 (DE3) recombinant Escherichia coli cells were pre-incubated as indicated, following addition of GS4B agarose beads for pull-down assay. 150 ng MBP and GST were used for negative control experiments. Immunoblotting was performed for OGT, His and GST. Lanes: 1, 2, 3 and 10, negative controls; 4 and 5, 150 ng GST-ECD and $500 \mathrm{ng}$ His-p120 were added to the system; 6-9, MBP-OGT was added in a dose gradient at $65 \mathrm{ng}, 165 \mathrm{ng}, 335 \mathrm{ng}, 500 \mathrm{ng}$, respectively. (B) CoIP assays in A549 cells were performed using anti-E-cadherin antibody. IgG was used as a negative control. Endogenous OGT, p120 and $\beta$-catenin co-precipitated with E-cadherin were immunoblotted using anti-OGT, anti-p120 and anti- $\beta$-catenin antibodies. ECD, E-cadherin cytoplasmic domain; OGT, O-linked $N$-acetylglucosamine transferase; H558A, mutant OGT; IgG, immunoglobulin G; CoIP, co-immunoprecipitation; GST, glutathione S-transferase; $\beta$-cat, $\beta$-catenin; Ecad, E-cadherin; MBP, maltose-binding protein.

E-cadherin endocytosis is regulated via complex mechanisms and depends on small $\mathrm{G}$ proteins, ubiquitination, cleavage events, the actomyosin cytoskeleton and other trans molecules in adherens junctions (22). These endocytic processes are required to be tightly regulated, as untimely de-stabilization of cadherin-mediated adhesion may modify the growth and architecture of tissues, resulting in pathological conditions, including cancer (23).

Association with p120 has been shown to be critical for the stability of E-cadherin-mediated adhesion. Multiple factors are involved in this stability control, including the masking of E-cadherin endocytic domains by p120 and the regulation of Rho pathway signaling (24). The armadillo repeat domain of p120 contains modular binding pockets, which are complementary to the hydrophobic and electrostatic properties of the JMD core of cadherin. Hence, p120 regulates the stability of cadherin by associating with the majority of its JMDs, subsequently masking residues implicated in clathrin-mediated endocytosis (dileucine motif) and Hakai-dependent ubiquitination of E-cadherin $(4,22)$.

It is well known that growth factor stimulation elevates the tyrosine phosphorylation of components of the E-cadherin cell adhesion complex, including p120, $\beta$-catenin and E-cadherin, which is accompanied by the endocytosis of E-cadherin (9). When the E-cadherin JMD was phosphorylated on tyrosine residues by tyrosine kinase, Hakai replaced p120 and bound to the E-cadherin JMD, inducing endocytosis of E-cadherin by ubiquitination of the latter (25). In addition, the phosphorylation of multiple serine and threonine residues in p120 was implicated in the regulation of E-cadherin function (26). In spite of the large number of studies available, the underlying mechanisms of the regulation of the dynamic interaction between p120 and E-cadherin have largely remained elusive.

$\mathrm{O}-\mathrm{GlcNAc}$ is known to the involved in the modification of $>1,000$ proteins involved in a wide range of biological processes, including transcription, signal transduction, metabolism and cell cycle progression (27). OGT and OGA as enzymes of the O-GlcNAc modification process are unique in terms of being encoded by single genes each, while enzymes involved in phosphorylation processes are encoded by several genes. To date, almost all available studies have highlighted the roles of OGT and OGA in the regulation O-GlcNAc cycling (12,14,28-31). However, OGT and OGA interact with thousands of target proteins, indicating that they may regulate protein functions independent of their enzyme activity (15). For example, it has been reported that OGT can bind to the host cell factor $\mathrm{C} 1$ (HCF-1) PRO repeat to $N$-acyl glycosylate the HCF-1N sub-unit as well as directly cleave the HCF-1 PRO repeat (32).

The present study found that OGT was able to directly bind to p120, although this complex was weak and unstable and the interaction was likely resolved by factors including surface-active agents in the cell lysate and kinetic factors during sample processing. Therefore, these samples were prepared as rapidly and gently as possible with low detergent concentrations of the cell lysates and handling on ice.

The results of the present study showed that wtOGT as well as the catalytically inactive OGT mutant H558A were able to decrease the levels of cytoskeleton-associated E-cadherin, p120 and $\beta$-catenin in H1299 cells, which was further confirmed in 4T1 cells. These results indicated that OGT is a negative regulator of the formation of the E-cadherin/catenin complex. 
A previous study by our group found that total E-cadherin was elevated following OGT silencing, while it was reduced by OGA inhibitors (12). In the present study, E-cadherin and p120 were assessed in the Triton X-100-insoluble or -soluble cell fractions. The results showed that E-cadherin levels in the Triton X-100-insoluble fraction were reduced following OGT overexpression and increased by OGT silencing, while E-cadherin levels in the Triton X-100-soluble fraction were not detectable or low and not markedly affected by OGT. As a multi-functional protein, p120 stabilizes cadherin-mediated cell-cell adhesion, releases Kaiso-mediated transcriptional suppression and maintains the Ras-related C3 botulinum toxin substrate 1/Cell division control protein 42/Ras homolog gene family, member A (RhoA) balance (33). The regulatory and armadillo domains of p120 are essential for its functions $(4,34)$. The present study demonstrated that OGT was able to bind to p120 and inhibit the interaction between p120 and E-cadherin. However, the present study did not investigate whether the binding of OGT to p120 may also affect the roles of RhoA and Kaiso. It has been reported that the activity of RhoA is regulated by O-GlcNAc in certain cell lines $(35,36)$, suggesting the likelihood of the hypothesized mechanism stated above. In addition, the activity of RhoA is an important regulatory factor for the E-cadherin adhesion complex (33), through which the binding of OGT to p120 may also affect E-cadherin-based cell-cell adhesion.

In conclusion, the present study revealed that OGT was able to inhibit the formation of the E-cadherin/catenin complex independent of its catalytic activity, and to reduce the interaction between p120 and E-cadherin by binding to p120. Together with the findings of previous studies indicating regulatory roles of OGT and global O-GlcNAc levels during development and cancer progression (12,29-31), the present study indicated that OGT inhibited E-cadherin-mediated cell-cell adhesion by O-GlcNAcylation as well as by directly binding to proteins relevant to the regulation of the E-cadherin adhesion complex, such as p120. OGT was indicated to effectively and adjustably exert its roles via several parallel mechanisms to regulate E-cadherin-mediated cell-cell adhesion in development and cancer progression.

\section{Acknowledgements}

The present study was supported by the National Natural Science Foundation of China (grant nos. 81172013, 81101505 and 81272264) and the Open Research Fund Program of the Key Laboratory of Marine Drugs [KLMD (OUC) no. 201308]. The authors would like to thank Professor Jin Won Cho (Department of Integrated OMICS for Biomedical Science, Yonsei University, Seoul, South Korea) for providing Flag-tagged human ncOGT expression vector, Professor Albert Reynolds (Vanderbilt University Medical Center, Nashville, TN, USA) for providing the LZRS-murine p120 1A-neo vector, Professor David J. Vocadlo (Department of Molecular Biology and Biochemistry, Simon Fraser University, Burnaby, BC, Canada) for providing the pMal-c2X-OGT vector and Professor Fred R. Miller (Department of Oncology, Barbara Ann Karmanos Cancer Institute, Wayne State University, Detroit, MI, USA) for providing 4T1 cells.

\section{References}

1. Gumbiner BM: Cell adhesion: The molecular basis of tissue architecture and morphogenesis. Cell 84: 345-357, 1996.

2. Jou TS, Stewart DB, Stappert J, Nelson WJ and Marrs JA: Genetic and biochemical dissection of protein linkages in the cadherin-catenin complex. Proc Natl Acad Sci U S A 92: 5067-5071, 1995.

3. Thoreson MA, Anastasiadis PZ, Daniel JM, Ireton RC, Wheelock MJ, Johnson KR, Hummingbird DK and Reynolds AB: Selective uncoupling of p120 (ctn) from E-cadherin disrupts strong adhesion. J Cell Biol 148: 189-202, 2000.

4. Ishiyama N, Lee SH, Liu S, Li GY, Smith MJ, Reichardt LF and Ikura M: Dynamic and static interactions between p120 catenin and E-cadherin regulate the stability of cell-cell adhesion. Cell 141: 117-128, 2010.

5. Nanes BA, Chiasson-Mackenzie C, Lowery AM, Ishiyama N, Faundez V, Ikura M, Vincent PA and Kowalczyk AP: p120-catenin binding masks an endocytic signal conserved in classical cadherins. J Cell Biol. 199: 365-80, 2012.

6. Ireton RC, Davis MA, van Hengel J, Mariner DJ, Barnes K, Thoreson MA, Anastasiadis PZ, Matrisian L, Bundy LM, Sealy L, et al: A novel role for p120 catenin in E-cadherin function. J Cell Biol 159: 465-476, 2002.

7. Peifer M and Yap AS: Traffic control: p120-catenin acts as a gatekeeper to control the fate of classical cadherins in mammalian cells. J Cell Biol 163: 437-440, 2003.

8. Keirsebilck A, Bonné S, Staes K, van Hengel J, Nollet F, Reynolds A and van Roy F: Molecular cloning of the human p120ctn catenin gene (CTNND1): Expression of multiple alternatively spliced isoforms. Genomics 50: 129-146, 1998.

9. Roura S, Miravet S, Piedra J, García de Herreros A and Duñach M: Regulation of E-cadherin/catenin association by tyrosine phosphorylation. J Biol Chem 274: 36734-36740, 1999.

10. Petrova YI, Spano MM and Gumbiner BM: Conformational epitopes at cadherin calcium-binding sites and p120-catenin phosphorylation regulate cell adhesion. Mol Biol Cell 23: 2092-2108, 2012.

11. Fukumoto Y, Shintani Y, Reynolds AB, Johnson KR and Wheelock MJ: The regulatory or phosphorylation domain of p120 catenin controls E-cadherin dynamics at the plasma membrane. Exp Cell Res 314: 52-67, 2008.

12. Gu Y, Mi W, Ge Y, Liu H, Fan Q, Han C, Yang J, Han F, Lu X and $\mathrm{Yu}$ W: GlcNAcylation plays an essential role in breast cancer metastasis. Cancer Res 70: 6344-6351, 2010.

13. Aslakson CJ and Miller FR: Selective events in the metastatic process defined by analysis of the sequential dissemination of subpopulations of a mouse mammary tumor. Cancer Res 52: 1399-1405, 1992.

14. Yang WH, Park SY, Nam HW, Kim do H, Kang JG, Kang ES, Kim YS, Lee HC, Kim KS and Cho JW: NFkappaB activation is associated with its O-GlcNAcylation state under hyperglycemic conditions. Proc Natl Acad Sci USA 105: 17345-17350, 2008.

15. Yuzwa SA, Yadav AK, Skorobogatko Y, Clark T, Vosseller K and Vocadlo DJ: Mapping O-GlcNAc modification sites on tau and generation of a site-specific O-GlcNAc tau antibody. Amino Acids 40: 857-868, 2011.

16. Zhu W, Leber B and Andrews DW: Cytoplasmic O-glycosylation prevents cell surface transport of E-cadherin during apoptosis. EMBO J 20: 5999-6007, 2001.

17. Martinez-Fleites C, Macauley MS, He Y, Shen DL, Vocadlo DJ and Davies GJ: Structure of an O-GlcNAc transferase homolog provides insight into intracellular glycosylation. Nat Struct Mol Biol 15: 764-765, 2008.

18. Pokutta S and Weis WI: Structure and mechanism of cadherins and catenins in cell-cell contacts. Annu Rev Cell Dev Biol 23: 237-261, 2007.

19. Takeichi M: Cadherins in cancer: Implications for invasion and metastasis. Curr Opin Cell Biol 5: 806-811, 1993.

20. Stepniak E, Radice GL and Vasioukhin V: Adhesive and signaling functions of cadherins and catenins in vertebrate development. Cold Spring Harb Perspect Biol 5: a002949, 2009.

21. Capaldo CT, Farkas AE and Nusrat A: Epithelial adhesive junctions. F1000Prime Rep 6: 1, 2014.

22. de Beco S, Amblard F and Coscoy S: New insights into the regulation of E-cadherin distribution by endocytosis. Int Rev Cell Mol Biol 295: 63-108, 2012.

23. Mosesson Y, Mills GB and Yarden Y: Derailed endocytosis: An emerging feature of cancer. Nat Rev Cancer 8: 835-850, 2008. 
24. Wheelock MJ, Shintani Y, Maeda M, Fukumoto Y and Johnson KR: Cadherin switching. J Cell Sci 121: 727-735, 2008.

25. Fujita Y, Krause G, Scheffner M, Zechner D, Leddy HE, Behrens J, Sommer T and Birchmeier W: Hakai, a c-Cbl-like protein, ubiquitinates and induces endocytosis of the E-cadherin complex. Nat Cell Biol 4: 222-231, 2002.

26. Alemà $S$ and Salvatore AM: p120 catenin and phosphorylation: Mechanisms and traits of an unresolved issue. Biochim Biophys Acta 1773: 47-58, 2007.

27. Hart GW, Slawson C, Ramirez-Correa G and Lagerlof O: Cross talk between O-GlcNAcylation and phosphorylation: Roles in signaling, transcription and chronic disease. Annu Rev Biochem 80: 825-858, 2011.

28. Wells L, Whelan SA and Hart GW: O-GlcNAc: A regulatory post-translational modification. Biochem Biophys Res Commun 302: 435-41, 2003.

29. Love DC, Krause MW and Hanover JA: O-GlcNAc cycling: Emerging roles in development and epigenetics. Semin Cell Dev Biol 21: 646-654, 2010.

30. Mi W, Gu Y, Han C, Liu H, Fan Q, Zhang X, Cong Q and Yu W: O GlcNAcylation is a novel regulator of lung and colon cancer malignancy. Biochim Biophys Acta 1812: 514-519, 2011
31. Liu Y, Li X, Yu Y, Shi J, Liang Z, Run X, Li Y, Dai CL, Grundke-Iqbal I, Iqbal K, et al: Developmental regulation of protein O-GlcNAcylation, O-GlcNAc transferase and O-GlcNAcase in mammalian brain. PLoS One 7: e43724, 2012.

32. Capotosti F, Guernier S, Lammers F, Waridel P, Cai Y, Jin J, Conaway JW, Conaway RC and Herr W: O-GlcNAc transferase catalyzes site-specific proteolysis of HCF-1. Cell 144: 376-388, 2011.

33. Pieters T, van Roy F and van Hengel J: Functions of p120ctn isoforms in cell-cell adhesion and intracellular signaling. Front Biosci (Landmark Ed) 17: 1669-1694, 2012.

34. Castaño J, Solanas G, Casagolda D, Raurell I, Villagrasa P, Bustelo XR, García de Herreros A and Duñach M: Specific phosphorylation of $\mathrm{p} 120$-catenin regulatory domain differently modulates its binding to RhoA. Mol Cell Biol 27: 1745-57, 2007.

35. Lima VV, Giachini FR, Carneiro FS, Carvalho MH, Fortes ZB, Webb RC and Tostes RC: O-GlcNAcylation contributes to the vascular effects of ET-1 via activation of the RhoA/Rho-kinase pathway. Cardiovasc Res 89: 614-622, 2011

36. Kneass ZT and Marchase RB: Protein O-GlcNAc modulates motility-associated signaling intermediates in neutrophils. J Biol Chem 280: 14579-14585, 2005. 